\title{
Observer Design for Gas Lifted Oil Wells
}

\author{
Ole Morten Aamo, Gisle Otto Eikrem, Hardy Siahaan, and Bjarne Foss
}

\begin{abstract}
Unstable regimes occuring for multiphase flow in vertical risers have successfully been stabilized using conventional linear control techniques. However, the control systems rely on downhole measurements which are at best unreliable, if at all available. In this paper, we design a nonlinear observer for the states of the multiphase flow that relies on topside measurements only, and apply it to estimate downhole pressure for feedback control. A key feature of the design is that it exploits the structure of the model to obtain robustness with respect to the internal flows in the system. The performance of the observer is demonstrated in simulations.
\end{abstract}

\section{INTRODUCTION}

The use of control in multiphase flow systems is an area of increasing interest for the oil and gas industry. Oil wells with highly oscillatory flow are a significant problem in the petroleum industry. Several different instability phenomena related to oil and gas wells exist, and in this study, unstable gas lifted wells will be the area of investigation. Gas lift is a technology to produce oil and gas from wells with low reservoir pressure by reducing the hydrostatic pressure in the tubing. Gas is injected into the tubing, as deep as possible, and mixes with the fluid from the reservoir, see Figure 1. The gas reduces the density of the fluid in the

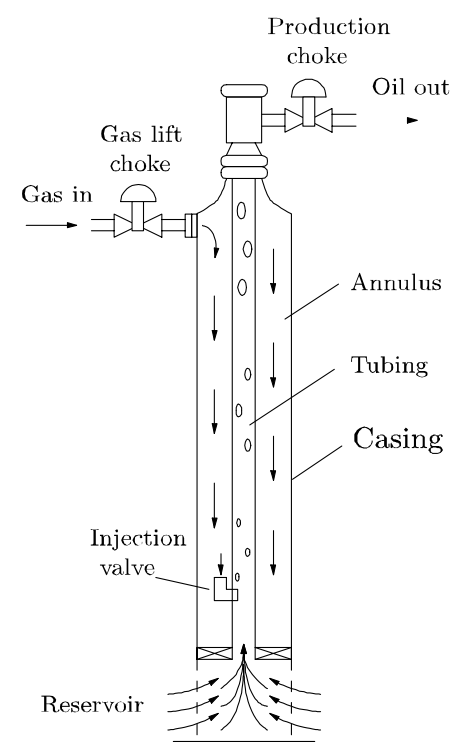

Fig. 1. A gas lifted oil well

tubing, which in turn reduces the downhole pressure (DHP),

This work was supported by the Gas Technology Center and Petronics at NTNU, and the Norwegian Research Council.

All authors are with the Department of Engineering Cybernetics, Norwegian University of Science and Technology, 7491 Trondheim, Norway. E-mail of corresponding author: aamoeitk. ntnu. no and thereby increases the production from the reservoir. The lift gas is routed from the surface and into the annulus, which is the volume between the casing and the tubing. The gas enters the tubing through a valve, or an injection orifice, which does not permit backflow from the tubing into the annulus. The dynamics of highly oscillatory flow in gas lifted wells can be described as follows:

1) Gas from the annulus starts to flow into the tubing. As gas enters the tubing the pressure in the tubing falls. This accelerates the inflow of gas.

2) The gas pushes the major part of the liquid out of the tubing.

3) Liquid in the tubing generates a blocking constraint downstream the injection orifice. Hence, the tubing gets filled with liquid and the annulus with gas.

4) When the pressure upstream of the injection orifice dominates the pressure on the downstream side, a new cycle starts.

For more information on this type of instability, often termed severe slugging, see [9].

There are in principle two approaches to eliminate highly oscillating well flow in gas lifted wells: The first approach is to increase the pressure drop caused by friction either by increasing the gas flow rate, reducing the opening of the production choke, reducing the size of the gas lift valve, or decoupling the dynamics of the annulus and tubing by obtaining supercritical flow through the injection valve; the second method is the use of active control to stabilize the well flow, which is the subject of this study. Figure 2 shows a conceptual gas lift production curve. The produced oil rate is a function of the flow rate of gas injected into the well. The curve shows under which conditions the well exhibits stable or highly oscillatory flow. It is important to note that the average production rate may be significantly lower with unstable, see the line labeled "open loop production", compared to stable well flow, see the line labeled "theoretical production". The region of optimum lift gas utilization may lie in the unstable region. In addition to causing lower oil production, large oscillations in the flow rate from the well causes poor downstream oil/water separation and flaring.

Stabilization of gas lifted wells using conventional control techniques has been studied for single well systems in [1], [6], [8], and for a two-well system in [3]. In [5], a state feedback control law was designed using Lyapunov theory, and the controller was used in an output feedback setting with an extended Kalman filter in [4]. In this paper, we design a nonlinear observer for the states of the multiphase flow in the tubing, and apply it to estimate 


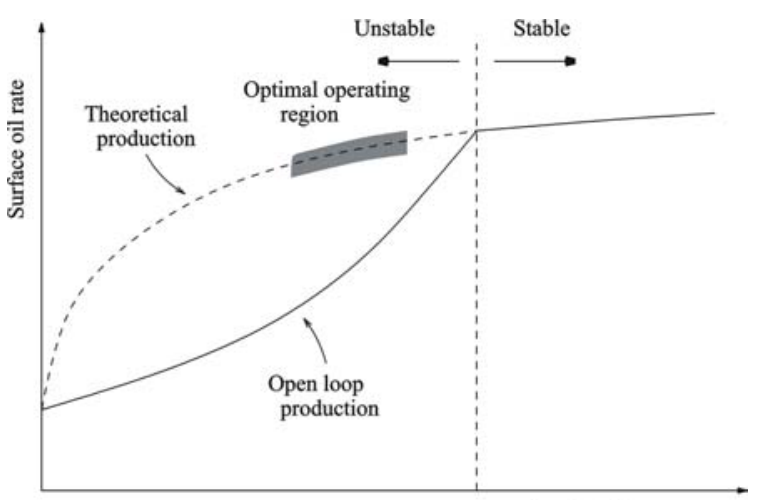

Gas injection rate

Fig. 2. The gas lift curve with the region of optimum lift gas utilization.

downhole pressure for feedback control. The design exploits the structure of the model to obtain robustness with respect to the internal flow between the annulus and the tubing.

The paper is organized as follows: In Section II we present a mathematical model of the gas lifted well due to [4], [5]; in Section III we design the observer and apply it in open-loop simulations, and; in Section IV the observer is applied for output feedback stabilization of the system. Concluding remarks are offered in Section V.

\section{Mathematical Model}

The process described in the introduction, and sketched in Figure 1, is modelled mathematically by three states: $x_{1}$ is the mass of gas in the annulus; $x_{2}$ is the mass of gas in the tubing, and; $x_{3}$ is the mass of oil in the tubing. Looking at Figure 1, we have

$$
\begin{aligned}
& \dot{x}_{1}=w_{g c}-w_{i v}, \\
& \dot{x}_{2}=w_{i v}-w_{p g}, \\
& \dot{x}_{3}=w_{r}-w_{p o},
\end{aligned}
$$

where $w_{g c}$ is a constant mass flow rate of lift gas into the annulus, $w_{i v}$ is the mass flow rate of lift gas from the annulus into the tubing, $w_{p g}$ is the mass flow rate of gas through the production choke, $w_{r}$ is the oil mass flow rate from the reservoir into the tubing, and $w_{p o}$ is the mass flow rate of produced oil through the production choke. The flows are modeled by

$$
\begin{aligned}
w_{g c} & =\text { constant flow rate of lift gas, } \\
w_{i v} & =C_{i v} \sqrt{\rho_{a, i} \max \left\{0, p_{a, i}-p_{t, i}\right\}}, \\
w_{p c} & =C_{p c} \sqrt{\rho_{m} \max \left\{0, p_{t}-p_{s}\right\}} u, \\
w_{p g} & =\frac{x_{2}}{x_{2}+x_{3}} w_{p c}, \\
w_{p o} & =\frac{x_{3}}{x_{2}+x_{3}} w_{p c}, \\
w_{r} & =C_{r}\left(p_{r}-p_{t, b}\right) .
\end{aligned}
$$

$C_{i v}, C_{p c}$, and $C_{r}$ are constants, $u$ is the production choke opening $(u(t) \in[0,1]), \rho_{a, i}$ is the density of gas in the annulus at the injection point, $\rho_{m}$ is the density of the oil/gas mixture at the top of the tubing, $p_{a, i}$ is the pressure in the annulus at the injection point, $p_{t, i}$ is the pressure in the tubing at the gas injection point, $p_{t}$ is the pressure at the top of the tubing, $p_{s}$ is the pressure in the separator, $p_{r}$ is the pressure in the reservoir, and $p_{t, b}$ is the pressure at the bottom of the tubing. The separator pressure, $p_{s}$, is assumed to be held constant by a control system, and the reservoir pressure, $p_{r}$, is assumed to be slowly varying and therefore treated as constant. Note that flow rates through the valves are restricted to be positive. The densities are modelled as follows

$$
\begin{aligned}
\rho_{a, i} & =\frac{M}{R T_{a}} p_{a, i}, \\
\rho_{m} & =\frac{x_{2}+x_{3}-\rho_{o} L_{r} A_{r}}{L_{t} A_{t}}
\end{aligned}
$$

and the pressures as follows

$$
\begin{aligned}
p_{a, i} & =\left(\frac{R T_{a}}{V_{a} M}+\frac{g L_{a}}{V_{a}}\right) x_{1}, \\
p_{t} & =\frac{R T_{t}}{M} \frac{x_{2}}{L_{t} A_{t}+L_{r} A_{r}-\nu_{o} x_{3}}, \\
p_{t, i} & =p_{t}+\frac{g}{A_{t}}\left(x_{2}+x_{3}-\rho_{o} L_{r} A_{r}\right), \\
p_{t, b} & =p_{t, i}+\rho_{o} g L_{r} .
\end{aligned}
$$

$M$ is the molar weight of the gas, $R$ is the gas constant, $T_{a}$ is the temperature in the annulus, $T_{t}$ is the temperature in the tubing, $V_{a}$ is the volume of the annulus, $V_{t}$ is the volume of the tubing, $L_{a}$ is the length of the annulus, $L_{t}$ is the length of the tubing, $A_{t}$ is the cross sectional area of the tubing above the injection point, $L_{r}$ is the length from the reservoir to the gas injection point, $A_{r}$ is the cross sectional area of the tubing below the injection point, $g$ is the gravity constant, $\rho_{o}$ is the density of the oil, and $\nu_{o}$ is the specific volume of the oil. The oil is considered incompressible, so $\rho_{o}$ is constant. The temperatures, $T_{a}$ and $T_{t}$ are slowly varying and therefore treated as constant.

We now assume that the multiphase flow through the production choke, that is, $w_{p c}$, is controlled by an inner loop flow controller that is fast compared to the dynamics of system (1)-(3). In other words, we may replace the control input $u$ (production choke opening), by the new control input $v=w_{p c}$ (flow rate through production choke), to obtain the system

$$
\begin{aligned}
& \dot{x}_{1}=w_{g c}-w_{i v}, \\
& \dot{x}_{2}=w_{i v}-\frac{x_{2}}{x_{2}+x_{3}} v, \\
& \dot{x}_{3}=w_{r}-\frac{x_{3}}{x_{2}+x_{3}} v .
\end{aligned}
$$

In the next section, we present our observer design, which is based on the system in the form (16)-(18).

\section{State Estimation}

In practice, measurements downhole in the tubing or annulus will in general not be available. If they are available, they must be considered unreliable due to the harsh 
conditions in which the sensors operate, and the fact that maintainance of the sensors is virtually impossible. Thus, we will in this work assume that we have measurements at the top of the annulus and tubing, only. The main challenge is how to deal with the multiphase flow in the tubing, whereas the single phase flow in the annulus can accurately be estimated based on one pressure measurement and one temperature measurement. Thus, we will assume that $x_{1}$ is measured. For estimation of the two remaining states, we measure the pressure at the top of the tubing. Our measurements are therefore

$$
y_{1}(t)=x_{1}(t) \text {, and } y_{2}(t)=p_{t}(t) .
$$

In addition, the variables of the fast flow controller governing the production choke, that is $v$ and $u$, are available.

\section{A. Reduced Order Observer Design}

Since the mass of gas in the annulus can be considered a measurement, we design a reduced order observer for the remaining two states. Before we state our main result, we state key assumptions and an intermediate result needed in the convergence proof for the observer. For definitions of control theoretic concepts used in this paper, see [7].

Assumption 1: The production choke is not allowed to close completely. That is,

$$
u \geq \delta_{u}>0, \forall t \geq 0
$$

Assumption 2: The states are bounded away from zero, and the part of the tubing below the gas injection point is filled with oil. More precisely,

$$
\left.\begin{array}{c}
x_{1} \geq \delta_{1}>0, x_{2} \geq \delta_{2}>0, \\
x_{3} \geq \rho_{o} L_{r} A_{r}+\delta_{3}>\rho_{o} L_{r} A_{r}
\end{array}\right\} \forall t \geq t_{0} .
$$

Assumption 3: The gas in the tubing has lower density than the oil. More precisely,

$$
L_{t} A_{t}+L_{r} A_{r}-\nu_{o}\left(x_{3}+x_{2}\right) \geq \delta_{g}>0, \forall t \geq 0 .
$$

Remark 4: Assumptions 1-3 are not restrictive. Since the production choke opening is a control input (from the fast flow controller), Assumption 1 can be satisfied by the construction of the control law. Of course, $\delta_{u}$ has consequences for the solvability of the state feedback regulation problem, and must therefore be sufficiently small. The first condition in (21) is satisfied for all $t>0$, and if $x_{1}\left(t_{0}\right) \neq 0$ for all $t \geq 0$. The second condition in (21) is imposed to deal with the fact that the model of the compressible multiphase flow in the tubing is invalid when the fluid is incompressible, which corresponds to $x_{2}=0$, since the pressure calculation is based on the ideal gas law. From a practical point of view, the flow in the tubing cannot forever stay single phase, since the pressure in the annulus would grow and gas would eventually penetrate into the tubing. The third condition in (21) states that the reservoir pressure must be high enough for oil to rise above the gas injection point in the tubing. The last assumption, Assumption 3, imposes an upper bound on the gas density in the tubing. For practical gas lifted oil wells, the density of gas will always be less than the density of oil under normal operation.
Lemma 5: Solutions of system (16)-(18) are bounded in the sense that there exists a constant $B$, depending on the initial state, such that

$$
x_{i} \leq B\left(x\left(t_{0}\right)\right), i=1,2,3, \forall t \geq 0 .
$$

In particular,

$$
x_{3}<\rho_{o}\left(L_{t} A_{t}+L_{r} A_{r}\right), \forall t \geq 0 .
$$

Proof: The condition (24) follows from incompressibility of the oil, and the fact that $x_{2}$ is strictly positive. Taking the Lyapunov function candidate $V=2 x_{1}+x_{2}+x_{3}$, it is straight forward to show that $\dot{V}$ is strictly negative for sufficiently large $V$. This defines a bounded region in state space which is globally attractive. $B$ can be taken to be the maximum value of the bound for this region and the norm of the initial state.

Theorem 6: Solutions $\hat{x}(t)=\left(\hat{x}_{2}(t), \hat{x}_{3}(t)\right)$ of the observer

$$
\begin{aligned}
\dot{\hat{z}}_{1}= & w_{g c}-\frac{\hat{z}_{1}-y_{1}}{\hat{z}_{2}-y_{1}} v+k_{1}\left(\hat{z}_{1}, \hat{z}_{2}, y_{1}, y_{2}\right) \\
\dot{\hat{z}}_{2}= & w_{g c}+C_{r}\left(p_{r}-\rho_{o} g L_{r}+\frac{A_{r}}{A_{t}} \rho_{o} g L_{r}+\frac{g}{A_{t}} y_{1}\right. \\
& \left.-y_{2}-\frac{g}{A_{t}} \hat{z}_{2}\right)-v+k_{2}\left(\hat{z}_{2}, u, v, y_{1}, y_{2}\right) \\
\hat{z}_{1} \geq & \delta_{2}+y_{1}, \text { and } \hat{z}_{2} \geq \rho_{o} L_{r} A_{r}+\delta_{3}+\hat{z}_{1} \\
\hat{x}_{2}= & \hat{z}_{1}-y_{1}, \\
\hat{x}_{3}= & \hat{z}_{2}-\hat{z}_{1},
\end{aligned}
$$

where the output injections, $k_{1}$ and $k_{2}$, are given by

$$
\begin{aligned}
& k_{1}\left(\hat{z}_{1}, \hat{z}_{2}, y_{1}, y_{2}\right)= \\
& c_{1}\left(\frac{M}{R T_{t}}\left(L_{t} A_{t}+L_{r} A_{r}-\nu_{o}\left(\hat{z}_{2}-\hat{z}_{1}\right)\right) y_{2}-\left(\hat{z}_{1}-y_{1}\right)\right), \\
& k_{2}\left(\hat{z}_{2}, u, v, y_{1}, y_{2}\right)= \\
& \quad c_{2}\left(\left(\frac{v}{C_{p c} u}\right)^{2}-\frac{\hat{z}_{2}-y_{1}-\rho_{o} L_{r} A_{r}}{L_{t} A_{t}}\left(y_{2}-p_{s}\right)\right),
\end{aligned}
$$

converge to the actual state $x(t)=\left(x_{2}(t), x_{3}(t)\right)$ exponentially fast in the following sense

$$
\|x(t)-\hat{x}(t)\| \leq C e^{-\gamma\left(t-t_{0}\right)},
$$

where $C$ depends on initial conditions, and

$$
\gamma=\min \left\{c_{1} \frac{\delta_{g}}{L_{t} A_{t}+L_{r} A_{r}}, \frac{C_{r} g}{A_{t}}+c_{2} \frac{\delta_{p}}{L_{t} A_{t}}\right\} .
$$

$\delta_{p} \geq 0$ is a constant satisfying $\max \left\{0, p_{t}-p_{s}\right\} \geq \delta_{p}$ for all $t \geq t_{0}$.

Proof: Define $z_{2}=x_{1}+x_{2}+x_{3}$, which is the total amount of mass in the system. From (16)-(18), (9), (15), and (14), its time derivative is

$$
\begin{aligned}
\dot{z}_{2}=w_{g c}+C_{r}\left(p_{r}-\rho_{o} g L_{r}\right. & +\frac{A_{r}}{A_{t}} \rho_{o} g L_{r} \\
+ & \left.\frac{g}{A_{t}} y_{1}-y_{2}-\frac{g}{A_{t}} z_{2}\right)-v .
\end{aligned}
$$


We estimate $z_{2}$ by $\hat{z}_{2}$, which is governed by

$$
\begin{aligned}
\dot{\hat{z}}_{2}=w_{g c}+ & C_{r}\left(p_{r}-\rho_{o} g L_{r}+\frac{A_{r}}{A_{t}} \rho_{o} g L_{r}\right. \\
+ & \left.\frac{g}{A_{t}} y_{1}-y_{2}-\frac{g}{A_{t}} \hat{z}_{2}\right)-v+k_{2}(\cdot),
\end{aligned}
$$

where $k_{2}(\cdot)$ is an output injection term to be determined. The observer error, $e_{2}=z_{2}-\hat{z}_{2}$, is governed by

$$
\dot{e}_{2}=-\frac{C_{r} g}{A_{t}} e_{2}-k_{2}(\cdot) \text {. }
$$

Take the Lyapunov function candidate $V_{2}=\frac{1}{2} e_{2}^{2}$. Its time derivative along solutions of (33) is

$$
\dot{V}_{2}=e_{2}\left(-\frac{C_{r} g}{A_{t}} e_{2}-k_{2}(\cdot)\right) \text {. }
$$

Selecting

$$
\begin{aligned}
k_{2}\left(\hat{z}_{2}, u, v, y_{1}, y_{2}\right)=c_{2}\left(\left(\frac{v}{C_{p c} u}\right)^{2}\right. & \\
- & \left.\frac{\hat{z}_{2}-y_{1}-\rho_{o} L_{r} A_{r}}{L_{t} A_{t}} \max \left\{0, y_{2}-p_{s}\right\}\right),
\end{aligned}
$$

where $c_{2}>0$, and inserting (35) into (34), we get

$$
\begin{aligned}
\dot{V}_{2}= & e_{2}\left(\left(-\frac{C_{r} g}{A_{t}} e_{2}\right)\right. \\
& -c_{2}\left(\frac{z_{2}-y_{1}-\rho_{o} L_{r} A_{r}}{L_{t} A_{t}} \max \left\{0, y_{2}-p_{s}\right\}\right. \\
& \left.\left.-\frac{\hat{z}_{2}-y_{1}-\rho_{o} L_{r} A_{r}}{L_{t} A_{t}} \max \left\{0, y_{2}-p_{s}\right\}\right)\right) \\
= & -\left(\frac{C_{r} g}{A_{t}}+c_{2} \frac{\max \left\{0, y_{2}-p_{s}\right\}}{L_{t} A_{t}}\right) e_{2}^{2} .
\end{aligned}
$$

So we obtain

$$
\left\|e_{2}(t)\right\| \leq\left\|e_{2}\left(t_{0}\right)\right\| e^{-\frac{C_{r} g}{A_{t}}\left(t-t_{0}\right)}
$$

Next, define $z_{1}=x_{1}+x_{2}$, which is the total mass of gas in the system. From (16)-(17), its time derivative is

$$
\dot{z}_{1}=w_{g c}-\frac{z_{1}-y_{1}}{z_{2}-y_{1}} v
$$

We estimate $z_{1}$ by $\hat{z}_{1}$, which is governed by

$$
\dot{\hat{z}}_{1}=w_{g c}-\frac{\hat{z}_{1}-y_{1}}{\hat{z}_{2}-y_{1}} v+k_{1}(\cdot),
$$

where $k_{1}(\cdot)$ is an output injection term to be determined. The observer error, $e_{1}=z_{1}-\hat{z}_{1}$, is governed by

$$
\dot{e}_{1}=-\frac{z_{1}-y_{1}}{z_{2}-y_{1}} v+\frac{\hat{z}_{1}-y_{1}}{\hat{z}_{2}-y_{1}} v-k_{1}(\cdot) .
$$

Notice that the observer error dynamics (33) and (40), is in a cascaded form, where the dynamics of $e_{2}$ is independent of $e_{1}$. Since $e_{2}$ converges to zero, we will seek to apply [2, Lemma 2]. Towards that end, we take the Lyapunov function candidate $V_{1}=\frac{1}{2} e_{1}^{2}$. Its time derivative along solutions of (40) is

$$
\begin{aligned}
\dot{V}_{1}= & e_{1}\left(-\frac{z_{1}-y_{1}}{z_{2}-y_{1}} v+\frac{\hat{z}_{1}-y_{1}}{\hat{z}_{2}-y_{1}} v-k_{1}(\cdot)\right) \\
= & e_{1}\left(-\frac{\left(\hat{z}_{2}-y_{1}\right) e_{1}-\left(\hat{z}_{1}-y_{1}\right) e_{2}}{\left(z_{2}-y_{1}\right)\left(\hat{z}_{2}-y_{1}\right)} v-k_{1}(\cdot)\right) \\
= & e_{1}\left(-\frac{v}{\left(z_{2}-y_{1}\right)} e_{1}+v \frac{\hat{z}_{1}-y_{1}}{\left(z_{2}-y_{1}\right)\left(\hat{z}_{2}-y_{1}\right)} e_{2}\right) \\
& -e_{1} k_{1}(\cdot) \\
= & -\frac{v}{z_{2}-y_{1}} e_{1}^{2} \\
& +v \frac{\hat{z}_{1}-y_{1}}{\left(z_{2}-y_{1}\right)\left(\hat{z}_{2}-y_{1}\right)} e_{1} e_{2}-e_{1} k_{1}(\cdot) .
\end{aligned}
$$

We now select

$$
\begin{aligned}
& k_{1}\left(\hat{z}_{1}, \hat{z}_{2}, y_{1}, y_{2}\right)= \\
& c_{1}\left(\frac{M}{R T_{t}}\left(L_{t} A_{t}+L_{r} A_{r}-\nu_{o}\left(\hat{z}_{2}-\hat{z}_{1}\right)\right) y_{2}-\hat{z}_{1}+y_{1}\right),
\end{aligned}
$$

where $c_{1}>0$, and obtain

$$
\begin{aligned}
\dot{V}_{1}= & -\frac{v}{z_{2}-y_{1}} e_{1}^{2}+v \frac{\hat{z}_{1}-y_{1}}{\left(z_{2}-y_{1}\right)\left(\hat{z}_{2}-y_{1}\right)} e_{1} e_{2} \\
& -c_{1} e_{1}\left(\frac{L_{t} A_{t}+L_{r} A_{r}-\nu_{o}\left(\hat{z}_{2}-\hat{z}_{1}\right)}{L_{t} A_{t}+L_{r} A_{r}-\nu_{o}\left(z_{2}-z_{1}\right)}\left(z_{1}-y_{1}\right)\right. \\
& \left.-\left(\hat{z}_{1}-y_{1}\right)\right) \\
= & -\left(\frac{v}{z_{2}-y_{1}}\right. \\
& \left.+c_{1} \frac{L_{t} A_{t}+L_{r} A_{r}-\nu_{o}\left(z_{2}-y_{1}\right)}{L_{t} A_{t}+L_{r} A_{r}-\nu_{o}\left(z_{2}-z_{1}\right)}\right) e_{1}^{2} \\
& +\left(v \frac{\hat{z}_{1}-y_{1}}{\left(z_{2}-y_{1}\right)\left(\hat{z}_{2}-y_{1}\right)}\right) e_{1} e_{2} \\
& -c_{1} \frac{\nu_{o}\left(z_{1}-y_{1}\right)}{L_{t} A_{t}+L_{r} A_{r}-\nu_{o}\left(z_{2}-z_{1}\right)}
\end{aligned}
$$

Using Lemma 5, Assumptions 2 and 3, and noticing that $\left(\hat{z}_{1}-y_{1}\right) /\left(\hat{z}_{2}-y_{1}\right)<1$, we obtain

$$
\begin{aligned}
\dot{V}_{1} \leq & -\left(\frac{v}{2 B}+c_{1} \frac{\delta_{g}}{L_{t} A_{t}+L_{r} A_{r}}\right) e_{1}^{2} \\
& +\left(\frac{v}{\delta_{2}+\delta_{3}}+c_{1} \frac{B \nu_{o}}{\delta_{g}}\right)\left\|e_{1}\right\|\left\|e_{2}\right\| .
\end{aligned}
$$

We can now apply [2, Lemma 2] with $V=\left(e_{1}^{2}+e_{2}^{2}\right) / 2$,

$$
\begin{gathered}
k_{3}=\min \left\{c_{1} \frac{\delta_{g}}{L_{t} A_{t}+L_{r} A_{r}}, \frac{C_{r} g}{A_{t}}+c_{2} \frac{\delta_{p}}{L_{t} A_{t}}\right\}, \\
g(\|e\|)=\left(\frac{v}{\delta_{2}+\delta_{3}}+c_{1} \frac{B \nu_{o}}{\delta_{g}}\right)\left\|e_{1}\right\|, \\
\sigma\left(\left\|e\left(t_{0}\right)\right\|, t-t_{0}\right)=\left\|e_{2}\left(t_{0}\right)\right\| e^{-\frac{C_{r} g}{A_{t}}\left(t-t_{0}\right)},
\end{gathered}
$$

to achieve the desired result, and in particular the estimate (31). 


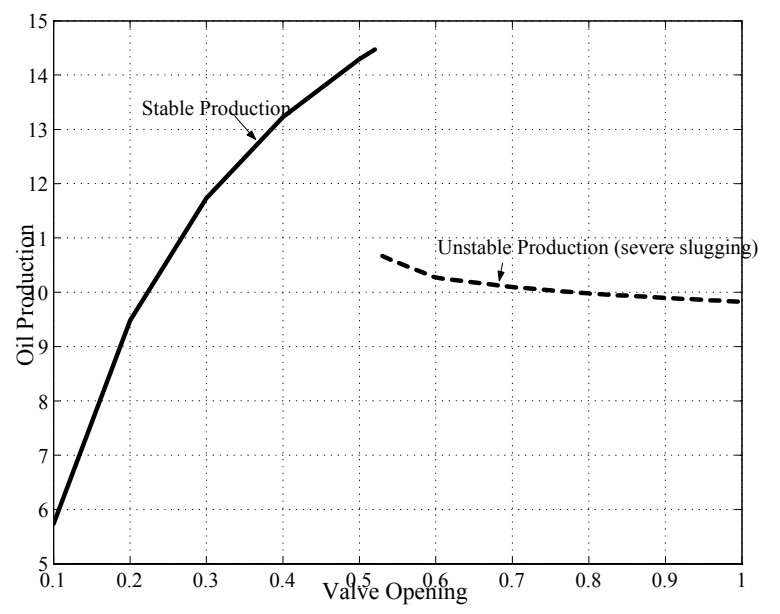

Fig. 3. Mean oil production versus production choke opening. Solid line indicates stable production and dashed line indicates severe slugging.

\section{B. Open-loop Simulations}

The numerical coefficients used in the simulations of system (16)-(18), are taken from a real full size gas lifted oil well. For these coefficients, simulations have been performed to calculate mean oil production as a function of production choke opening. The result is presented in Figure 3. The production is stable for small choke openings and increases as the choke opening is increased. At a choke opening of about 0.52 , the flow becomes unstable and goes into severe slugging, leading to a dramatic loss of production. The increasing trend of the production for small choke openings, suggests that a higher production is possible for large choke openings if the flow can be stabilized. This is shown to be the case in the next section. In this section, we will illustrate the performance of the observer by running open-loop simulations for the nominal case of perfect model. Figure 4 shows the states along with the estimates for the tubing over a six hour simulation with $c_{1}=0$ and $c_{2}=0$. The flow is clearly in the state of severe slugging, and the estimates converge to the actual states. It is clear that the convergence rate estimate $\gamma$, as defined in (31) is very conservative, since it is equal to 0 in the case shown in Figure 4. However, looking at inequality (44), the flow through the production choke, $v$, defines a better bound for the estimation convergence rate, and explains why the observer converges with $c_{1}=0$. Although $\gamma$ is a very conservative estimate for the convergence rate, (31) tells us that our observer can achieve any desired convergence rate by increasing $c_{1}$ and $c_{2}$. From the proof of Theorem 6 , we see that $c_{2}$ governs the convergence rate of the estimate for the total mass in the system (gas and oil), whereas $c_{1}$ governs the convergence rate of the estimate for the total mass of gas in the system, but with an upper bound governed by $c_{2}$. Simulations confirming faster convergence with increasing $c_{1}$ and $c_{2}$ have also been run, but are omitted here due to space limitations.

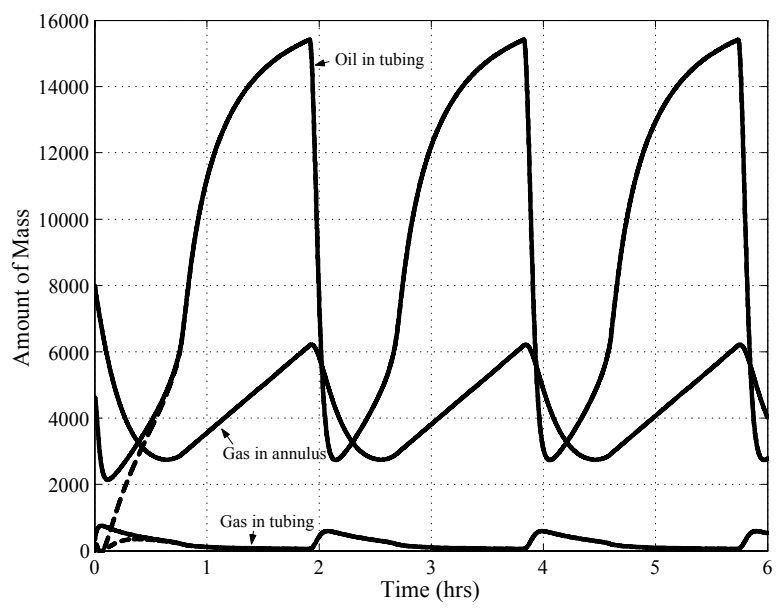

Fig. 4. States (solid line) and their estimates (dashed line) for the system during severe slugging.

\section{Anti-Slug Control by OUtput FeedBack}

\section{A. State Feedback Controller}

It has been shown in [3] that severe slugging can be attenuated by stabilizing the downhole pressure using a control law of the form

$$
v=v^{*}+K\left(p_{t, b}-p_{t, b}^{*}\right),
$$

where $v^{*}$ and $p_{t, b}^{*}$ are some appropriate constants. In [5], a state feedback design was presented that regulates the total mass of gas $\left(x_{1}+x_{2}\right)$ and total mass of oil $\left(x_{3}\right)$ to individual setpoints, $M_{g}$ and $M_{o}$, using the gas lift flow rate and the oil production flow rate as control inputs. In this demonstration, we will design a variant of this total mass controller. Having just one control input, we do not have the freedom to individually regulate the mass of gas and oil, but we may regulate the total mass, $M=x_{1}+x_{2}+x_{3}$. The resulting control law has the form

$$
v=w_{g c}+w_{r}+\lambda\left(M-M^{*}\right),
$$

where $M^{*}>0$ is the desired setpoint, and $\lambda$ is any positive number. Although (47) and (48) are designed with different objectives in mind, stabilizing downhole pressure and mass, respectively, their structure is in fact the same: As in (47), (48) also contains proportional feedback from the downhole pressure. The downhole pressure is in general not available as a measurement, and neither are the individual states in the tubing, but we may replace the states by their estimates generated by the observer designed in the previous section, to obtain the certainty equivalence controller. For linear systems, stability of the closed loop using the certainty equivalence controller is guaranteed by the separation principle of linear systems. For general nonlinear systems, however, not even an exponentially convergent observer in conjunction with an exponentially stabilizing state feedback control law can guarantee stability of the closed loop system. Thus, ignoring saturation in the control input, we 
argue in the next section that our closed-loop system is input-to-state stable with the observer error as input, so that stability is preserved under certainty equivalence control.

\section{B. A Nonlinear Separation Principle}

Consider the certainty equivalence controller

$$
v=w_{g c}+w_{r}\left(\hat{x}_{2}, \hat{x}_{3}\right)+\lambda\left(y_{1}+\hat{x}_{2}+\hat{x}_{3}-M^{*}\right) .
$$

Taking the Lyapunov function candidate

$$
V=\frac{1}{2}\left(M-M^{*}\right)^{2}
$$

we get

$$
\begin{aligned}
\dot{V}= & \left(M-M^{*}\right)\left(w_{r}\left(x_{2}, x_{3}\right)-w_{r}\left(\hat{x}_{2}, \hat{x}_{3}\right)\right. \\
& \left.-\lambda\left(y_{1}+\hat{x}_{2}+\hat{x}_{3}-M^{*}\right)\right) \\
= & -\lambda\left(M-M^{*}\right)^{2} \\
& +\left(M-M^{*}\right)\left(\lambda\left(e_{1}+e_{2}\right)+w_{r}\left(x_{2}, x_{3}\right)\right. \\
& \left.-w_{r}\left(x_{2}-e_{1}, x_{3}-e_{2}\right)\right)
\end{aligned}
$$

so that the closed loop system is input-to-state stable with

$$
\begin{aligned}
g\left(x_{2}, x_{3}, e_{1}, e_{2}\right)= & \lambda\left(e_{1}+e_{2}\right)+w_{r}\left(x_{2}, x_{3}\right) \\
& -w_{r}\left(x_{2}-e_{1}, x_{3}-e_{2}\right)
\end{aligned}
$$

as input. By the properties of our observer, $g(\cdot) \rightarrow 0$ as $t \rightarrow \infty$, and so $M \rightarrow M^{*}$ as $t \rightarrow \infty$. Having argued that $M \rightarrow M^{*}$ as $t \rightarrow \infty$, it remains to investigate the dynamics on the manifold defined by $M \equiv M^{*}$ in order to conclude that the state converges to some fixed point. Since the topic of this paper is observer design, we will not persue this further here, but refer the reader to [5].

\section{Closed-loop Simulations}

We complete this section by offering simulation results demonstrating the performance of our output feedback controller (49). Figure 5 shows that we have stabilized the flow at an oil production rate in excess of $16 \mathrm{~kg} / \mathrm{s}$, with a production choke opening of 0.8 . Recalling Figure 3, we see that this choke opening corresponds to unstable open loop production at $10 \mathrm{~kg} / \mathrm{s}$. Thus, the stabilized flow yields an oil production increase of $60 \%$ compared to the unstable open loop production at the same choke opening, and of about $11 \%$ compared to the upper performance limit for stable open loop production.

\section{Conclusions}

In this paper, we have designed a reduced order nonlinear observer for the states of the multiphase flow in the tubing. The observer relies on topside measurements, only. A key feature of the design is that it exploits the structure of the model to obtain robustness with respect to the internal flow between the annulus and the tubing. The performance of the observer was demonstrated in simulations.
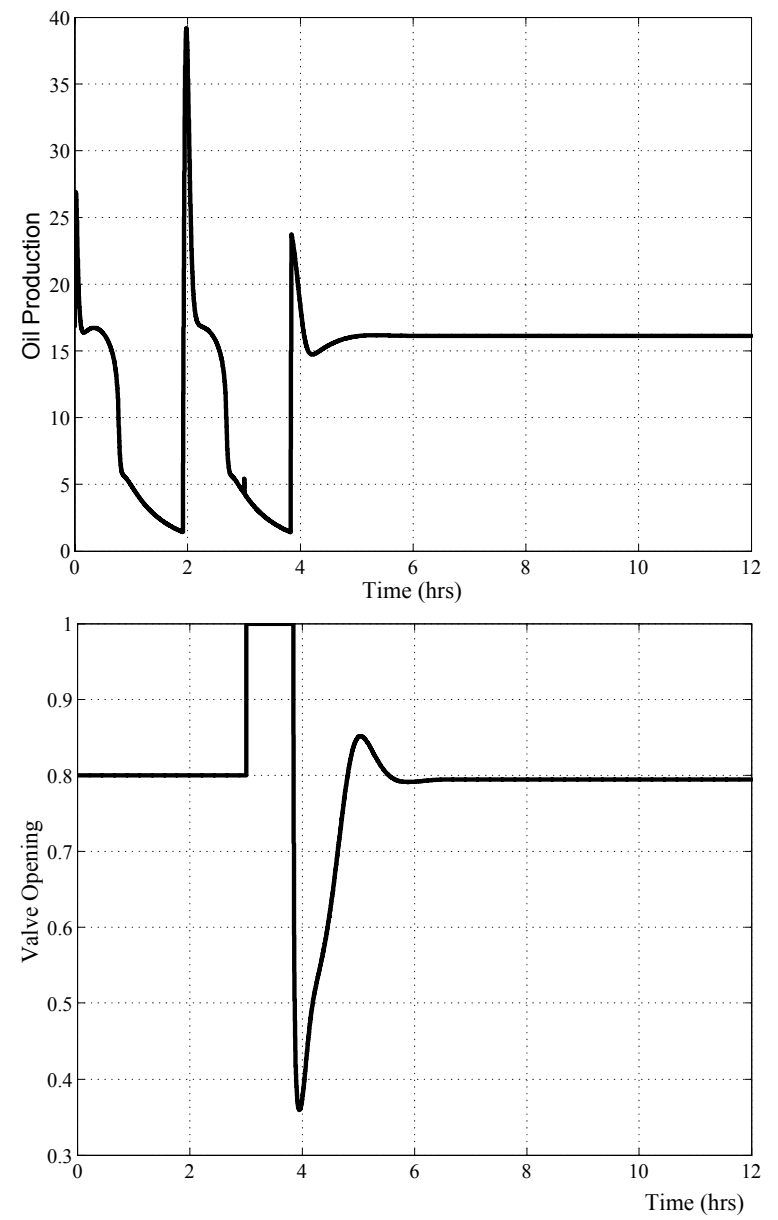

Fig. 5. Oil production and valve opening for output feedback control. Control is turned on at $t=3$ hours.

\section{ACKNOWLEDGEMENTS}

We thank Dr. Lars Imsland for valuable discussions.

\section{REFERENCES}

[1] M. Dalsmo, E. Halvorsen, and O. Slupphaug, "Active feedback control of unstable wells at the Brage field," SPE paper no. 77650.

[2] O.M. Aamo, M. Arcak, T.I. Fossen, and P.V. Kokotovic, "Global output tracking control of a class of Euler-Lagrange systems with monotonic non-linearities in the velocities," International Journal of Control, vol. 74, no. 7, pp. 649-658, 2001.

[3] G.O. Eikrem, B. Foss, L. Imsland, B. Hu, and M. Golan, "Stabilization of gas lifted wells," Proceedings of the 15th IFAC World Congress, Barcelona, Spain, 2002.

[4] G.O. Eikrem, L. Imsland, and B. Foss, "Stabilization of gas lifted wells based on state estimation," Proceedings of the International Symposium on Advanced Control of Chemical Processes, Hong Kong, China, 2004.

[5] L. Imsland, Topics in Nonlinear Control: Output Feedback Stabilization and Control of Positive Systems, Ph.D. thesis, NTNU, 2002.

[6] B. Jansen, M. Dalsmo, L. Nøkleberg, K. Havre, V. Kristiansen, and P. Lemetayer, "Automatic control of unstable gas lifted wells," SPE paper no. 56832.

[7] H. K. Khalil, Nonlinear Systems, 3rd Edition, Prentice-Hall, 2002.

[8] W.J.G.J. Kinderen and C.L. Dunham, "Real-time artificial lift optimization," SPE paper no. 49463.

[9] Z.G. Xu and M. Golan, "Criteria for operation stability of gas lift," SPE paper no. 19362. 